\title{
Implementation of Simple Multi Attribute Rating Technique Method using Decision Support System Concept (Case Recommendation of Salon Place in Pematangsiantar City)
}

\author{
Indra Riyana Rahadjeng ${ }^{1}$, Agus Perdana Windarto ${ }^{2}$ \\ ${ }^{1}$ Universitas Bina Sarana Informatika, Indonesia \\ ${ }^{2}$ STIKOM Tunas Bangsa, Pematangsiantar, North Sumatra, Indonesia \\ agus.perdana@amiktunasbangsa.ac.id
}

\begin{abstract}
The purpose of the research is to determine the ideal salon place considering the salon is a place visited by women to beautify themselves. This research can also be used as recommendations in the form of information in deciding whether consumers visit the salon or not based on research results. The method used in decision making is the SMART (Simple Multi Attribute Rating Technique) method. To determine the evaluation of the salon, researchers used 5 assessment criteria, among others: service (C1), facilities $(C 2)$, products (C3), price (C4) and Purchase Decision (C5). For alternatives used as a salon, among others: salon Sukim (A1), Necis (A2), Endah (A3), Crown (A4) and Chan Dhai (A5). The results obtained by Sukim (A1) with a final value of 0.8835 as the first rank, Endah Salon (A2) with a final score of 0.5530 as the second rank and Chan Dai (A5) with a final value of 0.45 as the third rank.
\end{abstract}

Keywords: Decision Support System, SMART Method, Salon Place, Ranking.

\section{Introduction}

Salon is a place that is often visited by women as a place to beautify themselves. Considered consumers are spoiled and given satisfying services without making consumers bother. Most consumers use the salon as a place to beautify and beautify themselves, with good and clean treatments such as: facials, cream baths, hair spas, body messages, steam, menicure and pedicure. This has become a trend and much in demand by consumers. Researchers are interested in recommending the ideal salon in the town of Pematangsiantar based on consumer choice. This needs to be done considering that every individual needs refeshing. One of them is Salon. It is hoped that this research can provide input to consumers, especially pematangsiantar community in choosing destinations to pamper themselves in accordance with consumer criteria. In this case the researchers used a questionnaire / questionnaire in evaluating the ideal salon. In this case a decision support system is needed to rank the ideal salon recommendations so that a decision support system is a solution offered to resolve the problem.

Many branches of computer science can solve complex problems. Among them is Artificial Intelligence [1]-[10]. Decision support systems are part of artificial intelligence that can be used to avoid the assessment of subjectivity that is produced [11]. Objectivity assessment is needed so that the decision support system can help consumers in choosing the ideal salon with consumer needs. Researchers used the SMART (Simple Multi Attribute Rating Technique) method. This is because the SMART method is able to solve problems with multi criteria. The following are some of the previous studies that used the SMART method to solve problems. One of them is done [11] with the title selection of cattle for beef cattle breeders using the SMART method. The results of the study explained that the SMART method can be applied to the selection of cattle types with the result that the Lemosin cattle (A1) 
type is the first recommendation with a final score of 1 and Bali cattle (A3) as the second recommendation with a final value of 0.702543 .

\section{Research Methodology}

\subsection{Decision Support System}

Decision support system is an application of information systems aimed at assisting leaders in the decision making process [12]. There are stages that must be carried out in the Decision Making System process, among others: Understanding Phase, Design Phase and Selection Phase [12]-[15].

\subsection{SMART Method}

SMART (Simple Multi Attribute Rating Technique) is a multi-criteria decision making technique based on the theory that each alternative consists of a number of criteria that have values and weights that illustrate how important compared to other criteria [16], [17].

\subsection{Data source}

This section explains how SMART stages and processes determine the ideal salon place in Pematangsiantar. This study uses 5 assessment criteria, including: Services (C1), Facilities (C2). Products (C3), Prices (C4) and Purchasing Decisions (C5). Alternatives used in the study include 5 best salons according to consumers in the city of Pematangsiantar through a questionnaire / questionnaire given to 150 respondents. The list of alternative salons includes: Sukin (A1), Necis Salon (A2), Endah (A3), Mahkota (A4) and Chan Dai (A5). The following is the normalized questionnaire data:

Table 1. Normalized Data

\begin{tabular}{|l|l|l|l|l|l|}
\hline \multirow{2}{*}{ Alternatif } & \multicolumn{5}{|c|}{ Kriteria } \\
\cline { 2 - 6 } & C1 & C2 & C3 & C4 & C5 \\
\hline Sukim & 0.81 & 0.62 & 0.77 & 0.68 & 0.56 \\
\hline Necis & 0.74 & 0.76 & 0.72 & 0.67 & 0.63 \\
\hline Endah & 0.77 & 0.50 & 0.69 & 0.62 & 0.68 \\
\hline Mahkota & 0.73 & 0.51 & 0.59 & 0.61 & 0.67 \\
\hline Chan Dhai & 0.72 & 0.60 & 0.76 & 0.69 & 0.57 \\
\hline Max & $\mathbf{0 . 8 1}$ & $\mathbf{0 . 7 6}$ & $\mathbf{0 . 7 7}$ & $\mathbf{0 . 6 9}$ & $\mathbf{0 . 6 8}$ \\
\hline Min & $\mathbf{0 . 7 2}$ & $\mathbf{0 . 5 0}$ & $\mathbf{0 . 5 9}$ & $\mathbf{0 . 6 1}$ & $\mathbf{0 . 5 6}$ \\
\hline
\end{tabular}

Information :

$\mathrm{C} 1$ : Service (weight $=45 \%)$

C2: Facilities (weight $=10 \%)$

C3: Product (weight $=25 \%)$

C4: Price (weight $=15 \%$ )

C5: Purchase decision (weight $=5 \%$ )

\section{Results and Discussion}

Here are the results of calculations in recommending the ideal salon place using the SMART method.

a) Give criteria weights with a value of 100-1 based on the importance of the criteria. The value given to the weight of this criterion is based on the judgment of the decision maker.

Table 2. Criteria Weight Value

\begin{tabular}{|l|l|}
\hline Criteria & Weight \\
\hline Service & 0.45 \\
\hline
\end{tabular}




\begin{tabular}{|l|l|}
\hline Criteria & Weight \\
\hline Facilities & 0.1 \\
\hline Product & 0.25 \\
\hline Price & 0.15 \\
\hline Purchase decision & 0.05 \\
\hline Total & $\mathbf{1}$ \\
\hline
\end{tabular}

b) Normalization of Criteria Weight

Table 3. Criteria Weight Normalization

\begin{tabular}{|l|l|l|}
\hline Criteria & Criteria Weight $\left(\mathbf{w}_{\mathbf{j}}\right)$ & Normalization of Criteria Weight \\
\hline Service & 0.45 & $0.45 / 1=0.45$ \\
\hline Facilities & 0.1 & $0.1 / 1=0.1$ \\
\hline Product & 0.25 & $0.25 / 1=0.25$ \\
\hline Price & 0.15 & $0.15 / 1=0.15$ \\
\hline Purchase decision & 0.05 & $0.05 / 1=0.05$ \\
\hline
\end{tabular}

c) Provide parameter values for each criterion. This step is required for qualitative data, for example for quality criteria (Strongly Agree $(\mathrm{SS})=4$, Agree $(\mathrm{S})=3$, Doubt - Ragu $(\mathrm{RR})=2$, Disagree $(\mathrm{TS})=1)$. In this study, the value for each criterion is in the form of quantitative so that no parameter values are needed anymore.

d) Determine the utility value. The value of this utility depends on the nature of each criterion. Utility value for each criterion.

Table 4. Determining Utility Value

\begin{tabular}{|l|l|}
\hline Criteria & \multicolumn{1}{|c|}{ Nature of Criteria } \\
\hline Service & The sooner the better \\
\hline Facilities & More complete is better \\
\hline Product & More complete is better \\
\hline Price & Cheaper is better \\
\hline Purchase decision & The sooner the better \\
\hline
\end{tabular}

The following service values, facilities, products, prices, purchase decisions for each criterion:
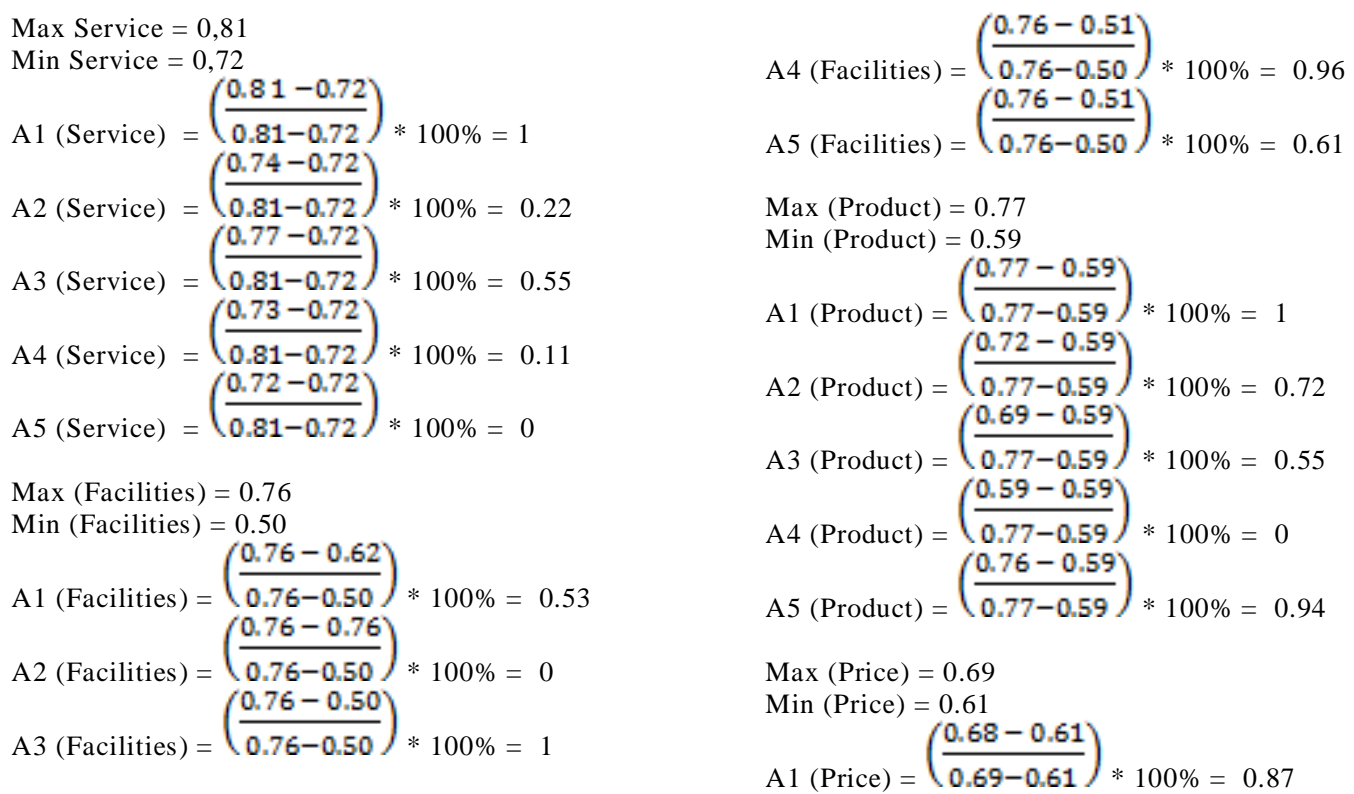


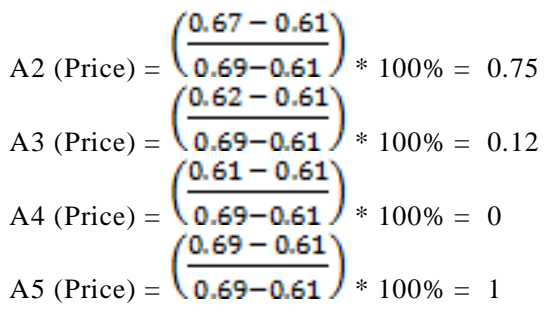

$\operatorname{Max}($ Purchase decision $)=0.68$ Min $($ Purchase decision $)=0.56$

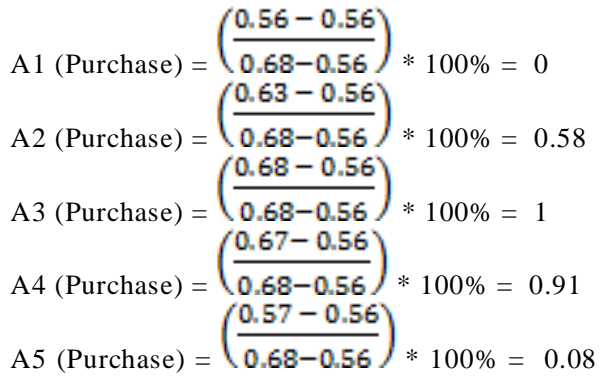

Following the complete results of the utility value calculation matrix can be seen in the following table:

Table 5. Alternative Value Utility Matrix F

\begin{tabular}{|l|l|l|l|l|l|}
\hline \multirow{2}{*}{ Alternative } & \multicolumn{6}{|c|}{ Citeria } \\
\cline { 2 - 6 } & \multicolumn{1}{|c|}{ C1 } & \multicolumn{1}{|c|}{ C2 } & \multicolumn{1}{|c|}{ C4 } & \multicolumn{1}{c|}{ C5 } \\
\hline Sukim & 1 & 0.53 & 1 & 0.87 & 0 \\
\hline Necis & 0.22 & 0 & 0.72 & 0.75 & 0.58 \\
\hline Endah & 0.55 & 1 & 0.55 & 0.12 & 1 \\
\hline Mahkota & 0.11 & 0.96 & 0 & 0 & 0.91 \\
\hline Can Dhai & 0 & 0.61 & 0.94 & 1 & 0.08 \\
\hline
\end{tabular}

e) Determine the final grade

Sukim - A1 (Service) $=1 * 0.45=0.45$

Sukim - A1 (Facilities) $=0.53 * 0.1=0.053$

Sukim - A1 (Product) $=1 * 0.25=0.25$

Sukim - A1 $($ Price $)=0.87 * 0.15=0.1305$

Sukim - A1 (Purchase decision) $=0 * 0.05=0$

Necis - A2 $($ Service $)=0.22 * 0.45=0.099$

Necis - A2 (Facilities) $=0 * 0.1=0$

Necis - A2 (Product) $=0.72 * 0.25=0.18$

Necis - A2 $($ Price $)=0.75 * 0.15=0.1125$

Necis - A2 $($ Purchase decision $)=0.58 * 0.05=0.029$

Endah - A3 $($ Service $)=0.55 * 0.45=0.2475$

Endah - A3 (Facilities) $=1 * 0.1=0.1$

Endah - A3 (Product) $=0.55 * 0.25=0.1375$

Endah - A3 $($ Price $)=0.12 * 0.15=0.18$

Endah - A3 (Purchase decision) $=1 * 0.05=0.05$

Mahkota - A4 $($ Service $)=0.1 * 0.45=0.045$

Mahkota - A4 (Facilities) $=0.96 * 0.1=0.096$

Mahkota - A4 (Product) $=0 * 0.25=0$

Mahkota $-\mathrm{A} 4$ (Price) $=0 * 0.15=0$

Mahkota - A4 $($ Purchase decision $)=0.91 * 0.05=0.045$

Chan Dhai - A5 (Service) $=0 * 0.45=0$

Chan Dhai - A5 (Facilities) $=0.61 * 0.1=0.061$

Chan Dhai - A5 (Product) $=0.94 * 0.25=0.235$

Chan Dhai - A5 (Price) $=1 * 0.15=0.15$

Chan Dhai - A5 (Purchase decision $)=0.08 * 0.05=0.004$ 
Following are the complete results of the SMART calculation method:

Table 6. Final Value Results

\begin{tabular}{|l|l|l|l|l|l|l|}
\hline \multirow{2}{*}{ Alt } & \multicolumn{5}{|c|}{ Criteria } & \multirow{2}{*}{$\begin{array}{c}\text { Score } \\
\text { End }\end{array}$} \\
\cline { 2 - 7 } & \multicolumn{1}{|c|}{ C1 } & \multicolumn{1}{c|}{ C2 } & \multicolumn{1}{c|}{ C3 } & \multicolumn{1}{c|}{ C5 } & \multicolumn{1}{c|}{ En } \\
\hline A1 & 0.45 & 0.053 & 0.25 & 0.1305 & 0 & 0.8835 \\
\hline A2 & 0.099 & 0 & 0.18 & 0.1125 & 0.029 & 0.4205 \\
\hline A3 & 0.2475 & 0.1 & 0.1375 & 0.018 & 0.05 & 0.553 \\
\hline A4 & 0.045 & 0.096 & 0 & 0 & 0.045 & 0.186 \\
\hline A5 & 0 & 0.061 & 0.235 & 0.15 & 0.004 & 0.45 \\
\hline
\end{tabular}

\section{Conclusion}

Based on the research results of the decision support system for selecting an ideal place in Pematangsiantar City using the SMART method, it can be concluded:

a) Recommendations for an ideal salon based on consumers in Pematangsiantar City based on 5 assessment criteria include: services, facilities, products, prices and purchasing decisions using the Simple Multi Attribute Rating Technique (SMART) method effective enough to be applied in determining the ideal salon place in Pematangsiantar City.

b) Based on the research results obtained Sukim (A1) with a final value of 0.8835 as the first rank, Endah Salon (A2) with a final value of 0.5530 as the second rank and Chan Dai (A5) with a final value of 0.45 as the third rank.

\section{References}

[1] C. Astria, A. P. Windarto, and Z. Musiafa, "Pemilihan Produk Sampo Sesuai Jenis Kulit Kepala Dengan Metode Promethee II," CESS (Journal Comput. Eng. Syst. Sci., vol. 4, no. 2, pp. 178-185, 2019.

[2] D. N. Batubara, D. R. S. P, and A. P. Windarto, "Penerapan Metode Promethee II Pada Pemilihan Situs Travel Berdasarkan Konsumen," J. SISFOKOM, vol. 8, no. 1, pp. 46-52, 2019.

[3] D. N. Batubara, A. P. Windarto, and M. R. Raharjo, "Penerapan Promethee II Pada Pemilihan Produk Conditioner Sebagai Upaya Peningkatan Minat Beli Konsumen," CESS (Journal Comput. Eng. Syst. Sci., vol. 4, no. 2, pp. 191-197, 2019.

[4] S. M. Dewi and A. P. Windarto, "Analisis Metode Electre Pada Pemilihan Usaha Kecil Home Industry Yang Tepat Bagi Mahasiswa," Sist. J. Sist. Inf., vol. 8, no. 3, pp. 377-385, 2019.

[5] C. Fadlan, A. P. Windarto, and I. S. Damanik, "Penerapan Metode MOORA pada Sistem Pemilihan Bibit Cabai ( Kasus : Desa Bandar Siantar Kecamatan Gunung Malela )," J. Appl. Informatics Comput., vol. 3, no. 2, pp. 42-46, 2019.

[6] B. Fachri, A. P. Windarto, and I. Parinduri, "Penerapan Backpropagation dan Analisis Sensitivitas pada Prediksi Indikator Terpenting Perusahaan Listrik," J. Edukasi dan Penelit. Inform., vol. 5, no. 2, pp. 202-208, 2019.

[7] D. R. S. P, A. A. Muin, and M. Amin, "Pemilihan Facial Wash Untuk Kulit Wajah Berminyak Dengan Metode Promethee II," CESS (Journal Comput. Eng. Syst. Sci., vol. 4, no. 2, pp. 222-229, 2019.

[8] A. P. Windarto and S. S, "Penerapan Algoritma Semut dalam Penentuan Distribusi Jalur Pipa Pengolahan Air Bersih,” J. Sist. Inf. Bisnis, vol. 2, pp. 123-132, 2018.

[9] D. A. Silitonga, M. Anjelita, and A. P. Windarto, "Fuzzy Inference System Untuk Prediksi Pembelian Bahan Bakar Pertamax Pada SPBU Di Kota Pematangsiantar," Syntax J. Inform., vol. 8, no. 2, pp. 75-83, 2019.

[10] I. Parlina, A. Wanto, and A. P. Windarto, "Artificial Neural Network Pada Industri Non Migas Sebagai Langkah Menuju Revolusi Industri 4.0," InfoTekJar J. Nas. Inform. dan Teknol. Jar., vol. 4, no. 1, pp. 155-160, 2019. 
[11] A. P. W. Gumilar Ramadhan Pangaribuan, W. P. Mustika, and A. Wanto, "Pemilihan Jenis Sapi bagi Peternak Sapi Potong dengan Metode SMART," Algoritm. J. Ilmu Komput. dan Inform., vol. 3, no. 1, pp. 30-37, 2019.

[12] S. R. Ningsih and A. P. Windarto, "Penerapan Metode Promethee II Pada Dosen Penerima Hibah P2M Internal," InfoTekJar (Jurnal Nas. Inform. dan Teknol. Jaringan), vol. 3, no. 1, pp. 20-25, 2018.

[13] A. Putrama and A. P. Windarto, "Analisis dalam menentukan produk bri syariah terbaik berdasarkan dana pihak ketiga menggunakan ahp," CESS (Journal Comput. Eng. Syst. Sci., vol. 3, no. 1, pp. 60-64, 2018.

[14] A. P. Windarto, "Penilaian Prestasi Kerja Karyawan PTPN III Pematangsiantar Dengan Metode Simple Additive Weighting (SAW)," J. Ris. Sist. Inf. Dan Tek. Inform., vol. 2, no. ISSN 2527-5771, pp. 84-95, 2017.

[15] C. Irwana, Z. F. Harahap, and A. P. Windarto, "SPK: Analisa Metode Moora Pada Warga Penerima Bantuan Renovasi RumaH,” JTI, vol. 10, no. 1, pp. 47-54, 2018.

[16] Faizal, F. Agus Setyaningsih, and M. Diponegoro, "Implementasi Sistem Pendukung Keputusan Dengan Metode SMART Untuk merangking Kemiskinan Dalam proses Penentuan Penerima Bantuan PKH," vol. 5, no. 2, pp. 13-24, 2017.

[17] Suryanto and M. Safrizal, "Sistem Pendukung Keputusan Pemilihan Karyawan Teladan dengan Metode SMART (Simple Multi Attribute Rating Technique)," J. CoreIT, vol. 1, no. 2, pp. 2460-738, 2015.

\section{Authors}

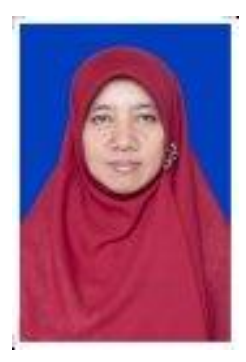

$\mathbf{1}^{\text {st }}$ Author

Indra Riyana Rahadjeng

Universitas Bina Sarana Informatika, Indonesia 